\title{
Delayed Retrograde Type A Aortic Dissection After Endografting of Descending Thoracic Aorta
}

\author{
Tiwari KK, ${ }^{1}$ Mariani $M,{ }^{2}$ Murzi $M,{ }^{2}$ Glauber $M^{2}$ \\ 'Sant Anna School for Higher Studies, Pisa, Italy, 'Department of Adult Cardiac Surgery, G. Pasquinucci Heart Hospital, \\ Massa, Italy
}

\begin{abstract}
We report a case of a delayed retrograde dissection occurred after endovascular repair of ruptured type B aortic dissection. A 75-year-old male, presented with acute hemothorax and aortic arch fissuration. He was successfully treated with descending aorta and aortic arch stenting. After six days, he developed retrograde type A dissection with aortic root involvement and aortic valve incompetence. The entire ascending aorta and the ventral part of the arch were successfully replaced by a composite graft; in addition, aortic valve was replaced with prosthesis. Patient had an uncomplicated course and radiologic follow-up at one month revealed a sealed fissuration and no signs of endoleak. Endovascular repair of acute ruptured aorta, although life saving may be associated with potentially lethal complications, and it requires close monitoring with prolonged hospital stay to recognize and treat promptly these fatal complications.
\end{abstract}

\section{INTRODUCTION}

Endovascular aortic repair (EVAR) of the descending thoracic aorta is an alternative to conventional open graft replacement. ${ }^{1,2}$ An increasing number of studies demonstrate that EVAR has as good result as surgical repair in treatment of the descending aorta aneurysm with fewer complications. ${ }^{3}$ EVAR is already the procedure of choice in patients with post traumatic aortic rupture; however, its application in complicated type B dissection is still controversial.Recently, a study demonstrated that EVAR intervention seems to be a promising option in patients with life-threatening acute aortic type $B$ dissection. ${ }^{4}$ Several complications of EVAR are reported, particularly in acute patients. ${ }^{5,6}$ We present a case of delayed retrograde type A dissection with root involvement after endovascular stenting of dissected descending thoracic aorta.

\section{CASE REPORT}

A 75-year-old patient was admitted to the emergency department with acute chest pain. He had presented with low blood pressure and reduced breath sounds on the left lower pulmonary area, blood sample revealed moderate anemia. Chest $X$ Ray revealed a left pleural effusion and an enlargement of the thoracic aorta profile. CT confirmed type B dissection with left sided hemothorax and a fissuration of the aortic arch anteriorly to the ostium of left subclavian artery (LSA). Entry tear was
Correspondence:
Dr. Kaushal K Tiwari,
G. Pasquinucci Heart Hospital
Via Aurelia Sud, Massa, Italy.
E-mail: drkaushalkł@yahoo.com 
located just distally to the LSA and the false lumen extended to the aortic bifurcation with good flow; all abdominal organs were well perfused (Figure 1a, b). Patient was stable and it was decided to cover the fissuration and the entry tear with an endovascular stent graft; a long stent graft (length $200 \mathrm{~mm}$ ) was placed in order to cover the distal part of the aortic arch and LSA with a free flow stent that crosses the ramification of the left carotid artery (LCA).

The angiographic and trans esophageal echocardiographic (TEE) control showed the presence of type IA leak in the ventral part of the arch, so we perform two balloon dilatation in the proximal part of the stent-graft was done. The final angiographic control showed no false lumen of dissection in the level of thoracic aorta, no leak, and good flow in the LCA. Post procedure TEE examination confirmed good positioning of the graft; proximal and distal landing zone and ascending aorta was inspected and no intimal tear or signs of dissection was revealed. Postoperative course was uneventful, and the patient recovered well. On the 6th post procedural day, transthoracic echocardiographic (TTE) control showed an intimal flap in the ascending aorta extending to the aortic root and a moderate to severe aortic regurgitation.

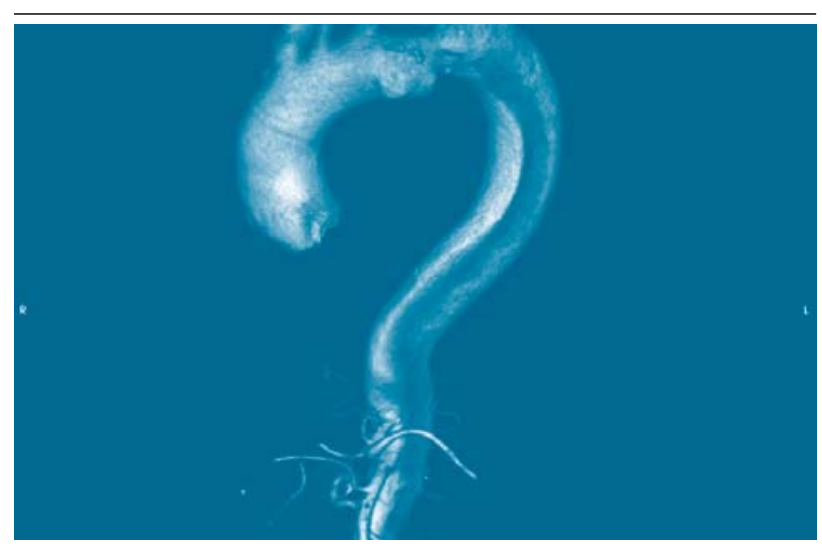

Figure 1a. Angio-CT at first admission 3D view showing type 2 Aortic dissection.

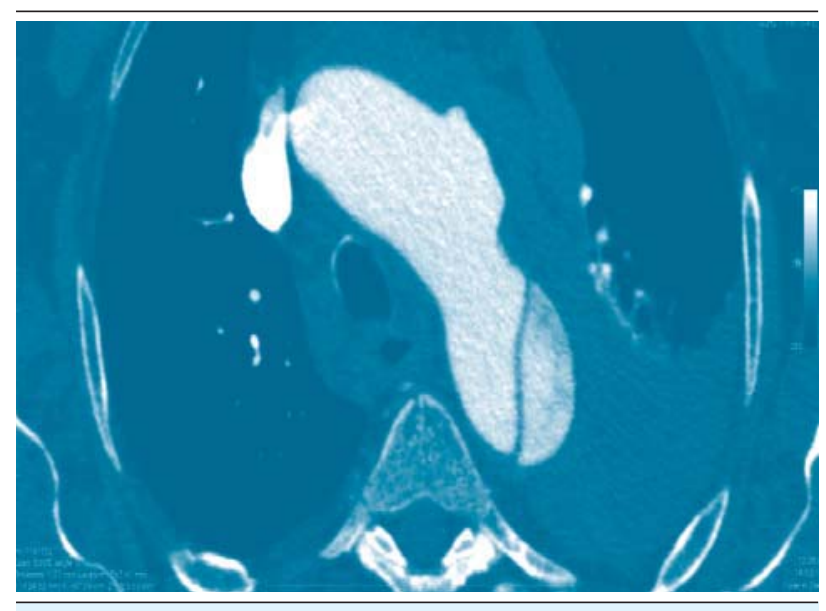

Fig $1 \mathrm{~b}$. Angio- CT at first admission 2D view.

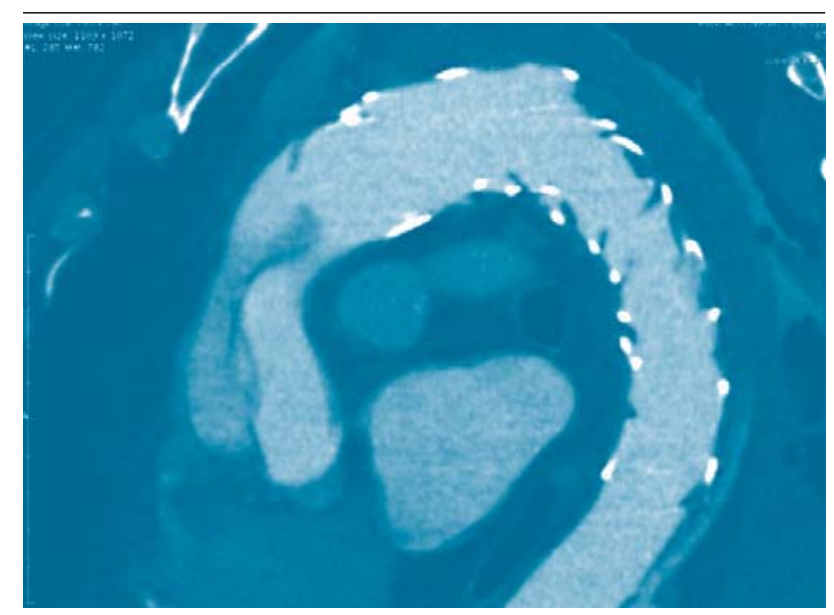

Fig 2. Angio-CT after retrograde dissection following stenting of the dissected aorta.

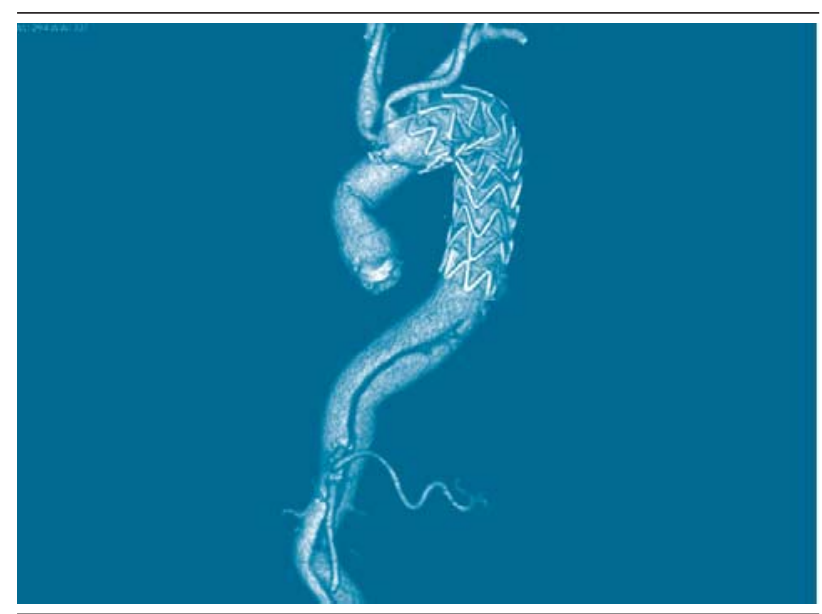

Fig 3. After surgical repair with Aortic pericardial Bioprosthesis

Additional computed tomography angiography (CTA) confirmed retrograde dissection of the arch and the ascending aorta involving the right coronary ostium with a partly thrombosed false lumen (Figure 2). We decided for open surgical intervention. During the surgery it was found that the intimal tear was located above the endoprosthesis near the anchoring strut in the ventral part of the aortic arch. Exploration of the endovascular graft revealed that it was mechanically intact and well positioned in the aortic arch. Apparently there was no communication between the two intimal tears.

The aortic valve, the ascending aorta, and the ventral portion of aortic arch were successfully replaced with a composite graft made of Aortic pericardial Bioprosthesis (Carpentier Edwards Magna No.23, USA) and tubular prosthesis (VascutecValsava No.26.). Postoperative course was uneventful. Post operative CT scan in intensive care unit revealed a good repair of the type A dissection, completely sealed fissure of the arch and no endoleak (Figure 3). Subsequently patient was discharged from 
hospital in eight days. Follow up CT scan at one year showed no negative change.

\section{DISCUSSION}

Studies on the use of EVAR in acute type B dissection are limited to few small series and usually they are conducted only for complicated cases. In IRAD registry ${ }^{1}$, type of treatment, conservative vs. invasive, did not differently affect the outcome of stable patients. In our reported case, there was an acute dissection leading to rupture of the aorta and we preferred to conduct EVAR instead of surgery because of higher mortality of surgical procedure in acutely ill patients with type B dissection. In the described case a very rare delayed acute type A dissection complicated the procedure. According to the chronology of the dissection, we can exclude that the dissection was present before the treatment because computed tomography and TEE has a $100 \%$ sensibility in excluding type A aortic dissection. Intraprocedural TEE monitoring and early post procedural angiography did not show any signs of dissection. Retrograde dissection is a rare complication of EVAR and is reported only in few cases. In one reported case, retrograde dissection occured after emergency treatment for ruptured type B acute dissection. $^{7}$ We can postulate that in this set of patients aortic wall strength, perhaps, is genetically reduced and may also be jeopardized by the acute event, so every manipulation of the aorta had a high risk of complication. Like other cases of delayed dissection, in our patient, intimal tear was located near the proximal anchoring strut in the aortic arch. Causative effect of the anchoring modulus of the device is almost out of doubts; in fact, spatial location of the intimal tear was very close to this one. Delayed adverse effect of endoaortic stent placement may be related to prolonged trauma on aortic wall by stent anchoring system. Another culprit could be the proximal balloon inflation to seal type I $A$ leak. Balloon inflation, could have caused a small lesion on the intima that could be complicated after some days of prolonged trauma by the stent strut. In fact, a small intimal tear in middle arch can be very difficult to visualize with intra-procedural TEE. Metallic stent graft may mask this area. In the acute phase blood pressure control may be very aggressive but in the recovery period, physical exertion and a less intensive pressure control may elicit that lesion of the aorta. Another issue is the stent graft sizing, ${ }^{8}$ in dissection a moderate over sizing of the diameter $(10-15 \%)$ is sufficient to achieve the stabilization without a major aortic wall stress. ${ }^{9}$ In our case, endoprosthesis oversizing was $10 \%$ of the diameter. Irrespective to the mechanism of the intimal tear and dissection, we believe that an extreme caution is mandatory during instrumentation of the aorta. In addition, if it is possible as advised by other observers, intra aortic balloon inflation should be avoided. We suggest also a prolonged post procedural in hospital stay with close monitoring of blood pressure and of the ascending aorta by TTE or if necessary by TEE. In our opinion, a pre discharge computed tomography is mandatory in all patients who had an acute aortic dissection and were treated by EVAR.

\section{REFERENCES}

1. Hagan PG, Nienaber CA, Isselbacher EM, Bruckman D, Karavite DJ, Russman PL et al. The International Registry of Acute Aortic Dissection (IRAD): new insights into an old disease. JAMA. 2000 Feb 16;283(7):897-903.

2. Nienaber CA, Spielmann RP, von Kodolitsch Y, Siglow V, Piepho A, Jaup T et al. Diagnosis of thoracic aortic dissection. Magnetic resonance imaging versus transesophageal echocardiography. Circulation. 1992 Feb;85(2):434-47.

3. Nienaber CA, Fattori R, Lund G, Dieckmann C, Wolf W, von Kodolitsch $Y$ et al. Nonsurgical reconstruction of thoracic aortic dissection by stent-graft placement. N Engl J Med. 1999 May 20;340(20):1539-45.

4. Duebener LF, Lorenzen P, Richardt G, Misfeld M, Nötzold A, Hartmann $\mathrm{F}$ et al. Emergency endovascular stent-grafting for life-threatening acute type B aortic dissections. Ann Thorac Surg. 2004 Oct;78(4):1261-6; discussion 1266-7.

5. Harris PL, Vallabhaneni SR, Desgranges P, Becquemin JP, van Marrewijk C, Laheij RJ. Incidence and risk factors of late rupture, conversion, and death after endovascular repair of infrarenal aortic aneurysms: the EUROSTAR experience. European
Collaborators on Stent/graft techniques for aortic aneurysm repair. J Vasc Surg. 2000 Oct;32(4):739-49.

6. Jacobowitz GR, Lee AM, Riles TS. Immediate and late explantation of endovascular aortic grafts: the endovascular technologies experience. J Vasc Surg. 1999 Feb;29(2):309-16.

7. Pasic M, Bergs P, Knollmann F, Zipfel B, Müller P, Hofmann $\mathrm{M}$ et al. Delayed retrograde aortic dissection after endovascular stenting of the descending thoracic aorta. J Vasc Surg. 2002 Jul;36(1):184-6.

8. Bortone AS, Schena S, Mannatrizio G, Paradiso V, Ferlan G, Dialetto $G$ et al. Endovascular stent-graft treatment for diseases of the descending thoracic aorta. Eur J Cardiothorac Surg. 2001 Sep;20(3):514-9.

9. Rubin S, Bayle A, Poncet A, Baehrel B. Retrograde aortic dissection after a stent graft repair of a type B dissection: how to improve the endovascular technique. Interact Cardiovasc Thorac Surg. 2006 Dec;5(6):746-8. 